PROCEEDINGS OF THE

AMERICAN MATHEMATICAL SOCIETY

Volume 139, Number 12, December 2011, Pages 4273-4284

S 0002-9939(2011)10869-4

Article electronically published on May 2, 2011

\title{
NONCANCELLATION FOR CONTRACTIBLE AFFINE THREEFOLDS
}

\author{
ADRIEN DUBOULOZ, LUCY MOSER-JAUSLIN, AND P.-M. POLONI
}

(Communicated by Lev Borisov)

\begin{abstract}
We construct two nonisomorphic contractible affine threefolds $X$ and $Y$ with the property that their cylinders $X \times \mathbb{A}^{1}$ and $Y \times \mathbb{A}^{1}$ are isomorphic, showing that the generalized Cancellation Problem has a negative answer in general for contractible affine threefolds. We also establish that $X$ and $Y$ are actually biholomorphic as complex analytic varieties, providing the first example of a pair of biholomorphic but not isomorphic exotic $\mathbb{A}^{3}$ 's.
\end{abstract}

\section{INTRODUCTION}

The Cancellation Problem asks if a complex algebraic variety $X$ of dimension $d$ such that $X \times \mathbb{A}^{n}$ is isomorphic to $\mathbb{A}^{n+d}$ is isomorphic to $\mathbb{A}^{d}$. This is a difficult problem in general and, apart form the trivial case $d=1$, an affirmative answer is known only in dimension 2 . One can ask more generally if two algebraic varieties $X$ and $Y$ such that $X \times \mathbb{A}^{n}$ is isomorphic to $Y \times \mathbb{A}^{n}$ for some $n \geq 1$ are isomorphic. This more general problem has an affirmative answer for a large class of varieties: intuitively, cancellation should hold provided that either $X$ or $Y$ does not contain too many rational curves. A precise characterization has been given by Iitaka and Fujita [1] in terms of logarithmic Kodaira dimension; namely, if either $X$ or $Y$, say $X$, has nonnegative logarithmic Kodaira dimension $\bar{\kappa}(X) \geq 0$, then every isomorphism between $X \times \mathbb{A}^{n}$ and $Y \times \mathbb{A}^{n}$ descends to an isomorphism between $X$ and $Y$. This assumption on the logarithmic Kodaira dimension turns out to be essential. Indeed, W. Danielewski 4 showed in 1989 that the rational affine surfaces $S_{1}=\left\{x y=z^{2}-1\right\}$ and $S_{2}=\left\{x^{2} y=z^{2}-1\right\}$ in $\mathbb{A}^{3}$ are nonisomorphic but have isomorphic cylinders $S_{1} \times \mathbb{A}^{1}$ and $S_{2} \times \mathbb{A}^{1}$. Since then, Danielewski's construction has been generalized and adapted to construct many new counterexamples of the same type, in arbitrary dimension [6, 8, 25].

However, all counterexamples constructed so far using variants of Danielewski's idea are remote from affine spaces: for instance, the Danielewski surfaces have nontrivial Picard groups and their underlying euclidean topological spaces are not contractible. Therefore, one may expect that cancellation holds for affine varieties close to the affine space. This is actually the case for smooth contractible or factorial surfaces. For the first ones, this follows from an algebro-geometric characterization of $\mathbb{A}^{2}$ due to Miyanishi-Sugie [18, 19, 24, which says that a smooth acyclic surface

Received by the editors June 30, 2010 and, in revised form, October 23, 2010.

2010 Mathematics Subject Classification. Primary 14R10.

(C)2011 American Mathematical Society Reverts to public domain 28 years from publication 
$S$ with $\bar{\kappa}(S)=-\infty$ is isomorphic to $\mathbb{A}^{2}$ (see also 3 for a purely algebraic selfcontained proof). On the other hand, the fact that generalized cancellation holds for smooth factorial affine surfaces $S$ seems to be folklore. Roughly, the argument goes as follows: first one may assume that $S$ has logarithmic Kodaira dimension $\bar{\kappa}=-\infty$. By virtue of a characterization due to T. Sugie [23, it follows that $S$ admits an $\mathbb{A}^{1}$-fibration $\pi: S \rightarrow C$ over a smooth curve $C$, that is, a surjective morphism with general fibers isomorphic to $\mathbb{A}^{1}$. The hypothesis that the Picard group of $S$ is trivial implies that the same holds for $C$, and so $C$ is a factorial affine curve. Combined with the classification of germs of degenerate fibers of $\mathbb{A}^{1}$ fibrations given by K.-H. Fieseler [8], the factoriality of $S$ implies that $\pi: S \rightarrow C$ has no degenerate fibers, whence is a locally trivial $\mathbb{A}^{1}$-bundle. Since $C$ is affine and factorial, $\pi: S \rightarrow C$ is actually a trivial $\mathbb{A}^{1}$-bundle $S \simeq C \times \mathbb{A}^{1} \rightarrow C$, and so the result follows from the affirmative answer to the generalized Cancellation Problem for curves due to Abhyankar-Eakin-Heinzer [1].

The situation turns out to be very different starting from dimension 3: D. Finston and S. Maubach 9] constructed 3-dimensional smooth factorial counterexamples to the generalized Cancellation Problem. The latter arise as total spaces of locally trivial $\mathbb{A}^{1}$-bundles over the complement of the isolated singularity of a Brieskorn surface $x^{p}+y^{q}+z^{r}=0$ in $\mathbb{A}^{3}$, with $1 / p+1 / q+1 / r<1$. By construction, these counterexamples are not contractible, having the homology type of a 3 -sphere. More recently, Z. Jelonek [12] found new factorial higher-dimensional counterexamples using affine varieties that admit stably trivial but nontrivial vector bundles. His constructions are reminiscent of M. Hochster's famous counterexample [10, which exploited the fact that the tangent bundle of the real 2-dimensional affine sphere is stably trivial but nontrivial. Since they come equipped with nontrivial vector bundles by construction, counterexamples obtained in this way are again remote from affine spaces.

The existence of contractible counterexamples in any dimension greater than or equal to 3 remained open. A famous candidate for being such a counterexample is the Russell cubic threefold $V$ defined by the equation $x^{2} y+z^{2}+t^{3}+x=0$ in $\mathbb{A}^{4}$. The latter is known to be contractible but not isomorphic to $\mathbb{A}^{3}$ (see e.g. [14] and [16]), and it is an open problem to decide whether or not $V \times \mathbb{A}^{1}$ is isomorphic to $\mathbb{A}^{4}$. In this article, we show that a mild variation on the above candidate already leads to contractible counterexamples to the generalized Cancellation Problem in dimension 3. Namely we consider the smooth affine threefolds

$$
X_{a}=\left\{x^{4} y+z^{2}+t^{3}+x+x^{2}+a x^{3}=0\right\}
$$

in $\mathbb{A}^{4}$, where $a$ is a complex parameter. We establish the following result:

Theorem. The threefolds $X_{a}$ are contractible, nonisomorphic to $\mathbb{A}^{3}$ and not isomorphic to each other. However, the cylinders $X_{a} \times \mathbb{A}^{1}, a \in \mathbb{C}$, are all isomorphic.

Recall that by virtue of a characterization due to A. Dimca [2, the varieties $X_{a}$, $a \in \mathbb{C}$, are all diffeomorphic to $\mathbb{R}^{6}$ when equipped with the euclidean topology and hence give examples of nonisomorphic exotic affine spaces. We show in contrast that they are all biholomorphic when considered as complex analytic manifolds. Actually, we establish the following stronger fact: the flat family

$$
W=\left\{x^{4} y+z^{2}+t^{3}+x+x^{2}+a x^{3}=0\right\} \subset \mathbb{A}^{4} \times \mathbb{A}^{1}
$$


of pairwise nonisomorphic threefolds $X_{a}$ parametrized by $\mathbb{A}^{1}=\operatorname{Spec}(\mathbb{C}[a])$ is holomorphically trivial, thus answering two open problems raised by M. Zaidenberg [27] and 29.

The article is organized as follows. In the first section, we consider more general contractible affine threefolds $X_{n, p}$ in $\mathbb{A}^{4}$ defined by equations of the form

$$
x^{n} y+z^{2}+t^{3}+x p(x)=0, \text { where } n \geq 2 \text { and } p(x) \in \mathbb{C}[x] .
$$

We provide, for each fixed integer $n \geq 2$, a complete classification of isomorphism classes of such varieties and their cylinders. As a corollary, we obtain that the varieties $X_{a}, a \in \mathbb{C}$, are pairwise nonisomorphic and have isomorphic cylinders. The second section is devoted to a geometric interpretation of the existence of an isomorphism between the cylinders $X_{a} \times \mathbb{A}^{1}, a \in \mathbb{C}$, in terms of a Danielewski fiber product trick construction.

\section{Main Results}

For any integer $n \geq 2$ and any polynomial $q \in \mathbb{C}[x, z, t]$, we consider the affine threefold $V_{n, q}$ in $\mathbb{A}^{4}=\operatorname{Spec}(\mathbb{C}[x, y, z, t])$ defined by the equation

$$
x^{n} y+z^{2}+t^{3}+x q(x, z, t)=0 .
$$

Note that $V_{n, q}$ is smooth if and only if $q(0,0,0)$ is a nonzero constant. The morphism $\pi=\operatorname{pr}_{x}: V_{n, q} \rightarrow \mathbb{A}^{1}$ is a flat $\mathbb{A}^{2}$-fibration restricting to a trivial $\mathbb{A}^{2}$ bundle over $\mathbb{A}^{1} \backslash\{0\}$ and with degenerate fiber $\pi^{-1}(0)$ isomorphic to the cylinder $\Gamma_{2,3} \times \mathbb{A}^{1}$ over the plane cuspidal curve $\Gamma_{2,3}=\left\{z^{2}+t^{3}=0\right\}$. This implies in particular that $V_{n, q}$ is factorial. Moreover, via the natural localization homomorphism, one may identify the coordinate ring of $V_{n, q}$ with the sub-algebra $\mathbb{C}\left[x, z, t, x^{-n}\left(z^{2}+t^{3}+x q(x, z, t)\right)\right]$ of $\mathbb{C}\left[x^{ \pm 1}, z, t\right]$. This says equivalently that $V_{n, q}$ is the affine modification $\sigma_{n, q}=\operatorname{pr}_{x, z, t}: V_{n, q} \rightarrow \mathbb{A}^{3}$ of $\mathbb{A}^{3}=\operatorname{Spec}(\mathbb{C}[x, z, t])$ with center at the closed subscheme $Z_{n, q}$ with defining ideal

$$
I_{n, q}=\left(x^{n}, z^{2}+t^{3}+x q(x, z, t)\right)
$$

and divisor $D=\left\{x^{n}=0\right\}$ in the sense of [14, that is, $V_{n, q}$ is isomorphic to the complement of the proper transform of $D$ in the blow-up of $\mathbb{A}^{3}$ with center at $Z_{n, q}$. It follows in particular from Theorem 3.1 in [14] that a smooth $V_{n, q}$ is contractible when considered as a complex manifold. When combined with a result due to P.M. Murthy [22, Theorem 3.6], this description also implies that every vector bundle on a smooth $V_{n, q}$ is trivial.

As a consequence of the general methods developed in [13, we have the following useful criterion to decide which threefolds $V_{n, q}$ are isomorphic.

Lemma 1.1. Every isomorphism $\Phi: V_{n_{1}, q_{1}} \stackrel{\sim}{\longrightarrow} V_{n_{2}, q_{2}}$ is the lift via $\sigma_{n_{1}, q_{1}}$ and $\sigma_{n_{2}, q_{2}}$ of an automorphism of $\mathbb{A}^{3}$ which maps the locus of the modification $\sigma_{n_{1}, q_{1}}$ isomorphically onto the one of the modification $\sigma_{n_{2}, q_{2}}$. Equivalently, there exists a commutative diagram

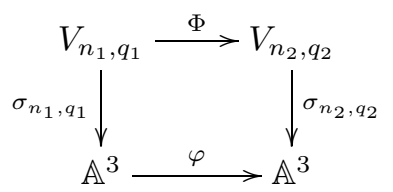

where $\varphi$ is an automorphism of $\mathbb{A}^{3}$ which preserves the hyperplane $\{x=0\}$ and maps $Z_{n_{1}, q_{1}}$ isomorphically onto $Z_{n_{2}, q_{2}}$. 
Proof. The fact that every automorphism of $\mathbb{A}^{3}$ satisfying the above property lifts to an isomorphism between $V_{n_{1}, q_{1}}$ and $V_{n_{2}, q_{2}}$ is an immediate consequence of the universal property of affine modifications, Proposition 2.1 in [14. For the converse we exploit two invariants of an affine variety $V$ : the Makar-Limanov invariant (resp. the Derksen invariant) of $V$ which is the sub-algebra ML $(V)$ (resp. Dk $(V)$ ) of $\Gamma\left(V, \mathcal{O}_{V}\right)$ generated by regular functions invariant under all (resp. at least one) nontrivial algebraic $\mathbb{G}_{a}$-actions on $V$ (see e.g. 28]). The same arguments as the ones used to treat the case of the Russell cubic threefold $V_{2,1}$ in [13, Theorem 9.1] show more generally that $\mathrm{ML}\left(V_{n, q}\right)=\mathbb{C}[x]$ and $\operatorname{Dk}\left(V_{n, q}\right)=\mathbb{C}[x, z, t]$ for every $q \in \mathbb{C}[x, z, t]$. This implies that any isomorphism between the coordinate rings of $V_{n_{1}, q_{1}}$ and $V_{n_{2}, q_{2}}$ restricts to an automorphism $\varphi^{*}$ of $\mathbb{C}[x, z, t]$ inducing one $x \mapsto a x+b$ of $\mathbb{C}[x]$, where $a \in \mathbb{C}^{*}$ and $b \in \mathbb{C}$. Actually, $b=0$ as the zero set of $a x+b$ in $V_{n_{i}, q_{i}}, i=1,2$, is singular if and only if $b=0$. So $\varphi^{*}$ stabilizes the ideal $(x)$. In turn, the fact that $I_{n_{i}, q_{i}}=x^{n_{i}} \Gamma\left(V_{n_{i}, q_{i}}, \mathcal{O}_{V_{n_{i}, q_{i}}}\right) \cap \mathbb{C}[x, z, t]$ implies that $\varphi^{*}\left(I_{n_{2}, q_{2}}\right)=I_{n_{1}, q_{1}}$. Now the assertion follows since the modification morphism $\sigma_{n, q}: V_{n, q} \rightarrow \mathbb{A}^{3}$ defined above is precisely the one induced by the natural inclusion of $\operatorname{Dk}\left(V_{n, q}\right)$ into $\Gamma\left(V_{n, q}, \mathcal{O}_{V_{n, q}}\right)$.

1.2. From now on, we only consider a very special case of smooth contractible threefolds $V_{n, q}$, namely, the $V_{n, p}$ defined by equations

$$
x^{n} y+z^{2}+t^{3}+x p(x)=0,
$$

where $p \in \mathbb{C}[x]$ is a polynomial such that $p(0) \neq 0$. We have the following result.

Theorem 1.3. For a fixed integer $n \geq 2$, the following hold:

(1) The algebraic varieties $V_{n, p_{1}}$ and $V_{n, p_{2}}$ are isomorphic if and only if there exists $\lambda, \varepsilon \in \mathbb{C}^{*}$ such that $p_{2}(x) \equiv \varepsilon p_{1}(\lambda x) \bmod x^{n-1}$.

(2) The cylinders $V_{n, p} \times \mathbb{A}^{1}$ are all isomorphic.

(3) The varieties $V_{n, p}$ are all biholomorphic as complex analytic manifolds.

Proof. Letting $r=z^{2}+t^{3}$, it follows from Lemma 1.1 above that $V_{n, p_{2}} \simeq V_{n, p_{1}}$ if and only if there exists an automorphism $\phi$ of $\mathbb{A}^{3}=\operatorname{Spec}(\mathbb{C}[x, z, t])$ which preserves the hyperplane $\{x=0\}$ and maps the closed subscheme with defining ideal $\left(x^{n}, r+x p_{2}(x)\right)$ isomorphically onto the one with defining ideal $\left(x^{n}, r+x p_{1}(x)\right)$. Since such an automorphism stabilizes the hyperplane $\{x=0\}$, there exists $\lambda \in \mathbb{C}^{*}$ such that $\phi^{*}(x)=\lambda x$. Furthermore, $\phi$ maps the curve $\Gamma_{2,3}=\left\{x=z^{2}+t^{3}=0\right\}$ isomorphically onto itself.

Therefore, there exists $\mu \in \mathbb{C}^{*}$ such that $\phi^{*} z \equiv \mu^{3} z \bmod x$ and $\phi^{*} t \equiv \mu^{2} t \bmod x$. Therefore, by composing $\phi$ with the automorphism $\theta: \mathbb{A}^{3} \stackrel{\sim}{\rightarrow} \mathbb{A}^{3},(x, z, t) \mapsto$ $\left(\lambda^{-1} x, \mu^{-3} z, \mu^{-2} t\right)$, we get an automorphism $\psi$ of $\mathbb{A}^{3}$ such that $\psi^{*} x=x$, $\psi^{*} z \equiv z \bmod x, \psi^{*} t \equiv t \bmod x$ and that maps the closed subscheme with defining ideal $\left(x^{n}, r+x p_{2}(x)\right)$ isomorphically onto the one with defining ideal $\left(x^{n}, r+\mu^{6} \lambda x p_{1}(\lambda x)\right)$. Letting $\varepsilon=\mu^{6} \lambda$, this implies $p_{2}(x) \equiv \varepsilon p_{1}(\lambda x) \bmod x^{n-1}$ by virtue of Lemma 1.4 below.

Conversely, if $p_{2}(x) \equiv \varepsilon p_{1}(\lambda x) \bmod x^{n-1}$, we let $\mu \in \mathbb{C}^{*}$ be such that $\varepsilon=\mu^{6} \lambda$. Then the automorphism

$$
(x, y, z, t) \mapsto\left(\lambda x, \lambda^{-n} \mu^{-6} y+\lambda^{-n} x^{-n+1}\left(\mu^{-6} p_{2}(x)-\lambda p_{1}(\lambda x)\right), \mu^{-3} z, \mu^{-2} t\right)
$$

of $\mathbb{A}^{4}$ maps $V_{n, p_{2}}$ isomorphically onto $V_{n, p_{1}}$. This proves (1). 
To prove (2) and (3), it is enough to show that for every $p \in \mathbb{C}[x]$ such that $p(0) \neq 0, V_{n, p}$ is biholomorphic to $V_{n, 1}$ and that these two threefolds have algebraically isomorphic cylinders. The arguments are similar to arguments developed in [21].

First, up to the composition by an isomorphism induced by an automorphism of $\mathbb{A}^{4}$ of the form $(x, y, z, t) \mapsto\left(\lambda x, \lambda^{-n} y, z, t\right)$, we may assume that $p(0)=1$. Notice also that the ideals $\left(x^{n}, z^{2}+t^{3}+x\right)$ and $\left(x^{n}, p(x)\left(z^{2}+t^{3}+x\right)\right)$ are equal. Therefore, by virtue of Lemma $1.1 V_{n, 1}$ is isomorphic as an algebraic variety to the variety $W_{n, p}$ defined by the equation $x^{n} y+p(x)\left(z^{2}+t^{3}+x\right)=0$.

Letting $f \in \mathbb{C}[x]$ be a polynomial such that $\exp (x f(x)) \equiv p(x) \bmod x^{n}$, one checks that the biholomorphism $\psi$ of $\mathbb{A}^{4}$ defined by

$$
\Psi(x, z, t)=\left(x, y-\frac{\exp (x f(x))-p(x)}{x^{n}}\left(z^{2}+t^{3}\right), \exp \left(\frac{1}{2} x f(x)\right) z, \exp \left(\frac{1}{3} x f(x)\right) t\right)
$$

maps $W_{n, p}$ onto $V_{n, p}$. So (3) follows.

For (2), we choose polynomials $g_{1} \in \mathbb{C}[x]$ such that $\exp \left(\frac{1}{2} x f(x)\right) \equiv g_{1} \bmod x^{n}$ and $g_{2} \in \mathbb{C}[x]$ relatively prime to $g_{1}$ such that $\exp \left(\frac{1}{3} x f(x)\right) \equiv g_{2} \bmod x^{n}$. Since $g_{1}(0)=g_{2}(0)=1$, the polynomials $x^{n} g_{1}, x^{n} g_{2}$ and $g_{1} g_{2}$ generate the unit ideal in $\mathbb{C}[x]$. Hence there exist polynomials $h_{1}, h_{2}, h_{3} \in \mathbb{C}[x]$ such that

$$
\left(\begin{array}{ccc}
g_{1} & 0 & x^{n} \\
0 & g_{2} & x^{n} \\
h_{1}(x) & h_{2}(x) & h_{3}(x)
\end{array}\right) \in \mathrm{GL}_{3}(\mathbb{C}[x]) .
$$

This matrix defines a $\mathbb{C}[x]$-automorphism of $\mathbb{C}[x][z, t, w]$ which maps the ideal $\left(x^{n}, r+x p(x)\right)$ of $\mathbb{C}[x][z, t, w]$ onto the one $\left(x^{n}, p(x)(r+x)\right)=\left(x^{n}, r+x\right)$. Since these ideals coincide with the centers of the affine modifications $\sigma_{n, p} \times$ id $: V_{n, p} \times$ $\mathbb{A}^{1} \rightarrow \mathbb{A}^{4}$ and $\sigma_{n, 1} \times$ id $: V_{n, 1} \times \mathbb{A}^{1} \rightarrow \mathbb{A}^{4}$ respectively, we can conclude by Proposition 2.1 in 14 that the corresponding automorphism of $\mathbb{A}^{4}=\operatorname{Spec}(\mathbb{C}[x, z, t, w])$ lifts to an isomorphism between $V_{n, 1} \times \mathbb{A}^{1}$ and $V_{n, p} \times \mathbb{A}^{1}$. This completes the proof.

Lemma 1.4. Let $n \geq 2$ and $p_{1}, p_{2} \in \mathbb{C}[x]$ be polynomials of degree $\leq n-2$. If there exists a $\mathbb{C}[x]$-automorphism $\Phi$ of $\mathbb{C}[x][z, t]$ such that $\Phi \equiv \mathrm{id} \bmod x$ and $\Phi\left(x^{n}, z^{2}+t^{3}+x p_{1}\right)=\left(x^{n}, z^{2}+t^{3}+x p_{2}\right)$, then $p_{1}=p_{2}$.

Proof. We let $r=z^{2}+t^{3}$ and also let $p_{i}=\sum_{k=0}^{n-2} a_{i k} x^{k}, i=1,2$. We let $n_{0} \geq 1$ be the largest integer such that $\Phi \equiv$ id $\bmod x^{n_{0}}$. If $n_{0} \geq n-1$, we are done. Otherwise, there exist $\alpha, \beta \in \mathbb{C}[x, z, t]$ such that $\Phi\left(r+x p_{1}\right)=\left(1+x^{n_{0}} \alpha\right)\left(r+x p_{2}\right)+x^{n} \beta$. Since the determinant of the Jacobian of $\Phi$ is a nonzero constant, there exists $h \in \mathbb{C}[z, t]$ such that

$$
\left\{\begin{array}{l}
\Phi(z) \equiv z+x^{n_{0}} \partial_{t} h \bmod x^{n_{0}+1} \\
\Phi(t) \equiv t-x^{n_{0}} \partial_{z} h \bmod x^{n_{0}+1}
\end{array}\right.
$$

It follows that $\Phi\left(r+x p_{1}\right) \equiv r+x p_{1}+x^{n_{0}} \operatorname{Jac}(r, h) \bmod x^{n_{0}+1}$. By comparing with the other expression for $\Phi\left(r+x p_{1}\right)$, we find that $p_{1} \equiv p_{2} \bmod x^{n_{0}-1}$ and $a_{1, n_{0}-1}+$ $\operatorname{Jac}(r, h)=\alpha(0, z, t) r+a_{2, n_{0}-1}$. Since $\operatorname{Jac}(r, h) \in(z, t) \mathbb{C}[z, t]$, we obtain $a_{1, n_{0}-1}=$ $a_{2, n_{0}-1}$ and $\operatorname{Jac}(r, h)=\alpha(0, z, t) r$. Moreover, the condition $\operatorname{Jac}(r, h) \in r \mathbb{C}[z, t]$ implies that $h=\gamma(z, t) r+c$ for some $\gamma \in \mathbb{C}[z, t]$ and $c \in \mathbb{C}$. Now we consider the 
exponential $\mathbb{C}[x] /\left(x^{n}\right)$-automorphism $\exp (\delta)$ of $\mathbb{C}[x] /\left(x^{n}\right)[z, t]$ associated with the Jacobian derivation

$$
\delta=x^{n_{0}} \operatorname{Jac}\left(\cdot, \gamma(z, t)\left(r+x p_{1}\right)\right) .
$$

Since the determinant of the Jacobian of $\exp (\delta)$ is equal to 1 (see [20]), it follows from [26] that there exists a $\mathbb{C}[x]$-automorphism $\Theta$ of $\mathbb{C}[x][z, t]$ such that $\Theta \equiv$ $\exp (\delta) \bmod x^{n}$. By construction, $\Theta \equiv \Phi \bmod x^{n_{0}+1}$ and, since $r+x p_{1}$ divides

$$
\delta\left(r+x p_{1}\right)=x^{n_{0}} \operatorname{Jac}\left(r+x p_{1}, \gamma(z, t)\left(r+x p_{1}\right)\right)=x^{n_{0}}\left(r+x p_{1}\right) \operatorname{Jac}\left(r+x p_{1}, \gamma(z, t)\right),
$$

$\Theta$ preserves the ideal $\left(x^{n}, r+x p_{1}\right)$. It follows that $\Psi=\Phi \circ \Theta^{-1}$ is a $\mathbb{C}[x]$ automorphism of $\mathbb{C}[x][z, t]$ such that $\Psi\left(x^{n}, z^{2}+t^{3}+x p_{1}\right)=\left(x^{n}, z^{2}+t^{3}+x p_{2}\right)$ and such that $\Psi \equiv \operatorname{id} \bmod x^{n_{0}+1}$. Now the assertion follows by induction.

Remark 1.5. In the proofs above, letting $Z_{n, p}$ be the centers of the affine modifications defining the threefolds $V_{n, p}$, the crucial point is to characterize the existence of isomorphisms between the $Z_{n, p}$ that are induced by automorphisms of the ambient space $\mathbb{A}^{3}$. Note that for a fixed integer $n \geq 2$, these closed subschemes $Z_{n, p}$ with defining ideals $I_{n, p}=\left(x^{n}, z^{2}+t^{3}+x p(x)\right)$ are all isomorphic as abstract schemes, even as abstract infinitesimal deformations of the plane cubic $\Gamma_{2,3}=\left\{z^{2}+t^{3}=0\right\}$ over Spec $\left(\mathbb{C}[x] /\left(x^{n}\right)\right)$. Indeed, letting $g_{1}(x), g_{2}(x) \in \mathbb{C}[x]$ be polynomials such that $\left(g_{1}(x)\right)^{2} \equiv p(x) \bmod x^{n}$ and $\left(g_{2}(x)\right)^{3} \equiv p(x) \bmod x^{n}$, one checks for instance that the automorphism $\xi$ of $\operatorname{Spec}\left(\mathbb{C}[x] /\left(x^{n}\right)[z, t]\right)$ defined by

$$
\xi(x, z, t)=\left(x, g_{1}(x) z, g_{2}(x) t\right)
$$

induces an isomorphism between $Z_{n, p}$ and $Z_{n, 1}$. However, Theorem 1.3 says in particular that no isomorphism of this kind can be lifted to an automorphism of $\mathbb{A}^{3}=\operatorname{Spec}(\mathbb{C}[x, z, t])$. In other words, the $Z_{n, p}$ 's can be considered as defining nonequivalent closed embeddings of $Z_{n, 1}$ in $\mathbb{A}^{3}$.

The above proof also gives counterexamples, in dimension four, to the so-called stable equivalence problem (see [17] and 21]). Indeed, it implies that for each $n \geq 2$ and each $p(x) \in \mathbb{C}[x]$ with $p(0) \neq 0$, the polynomials $x^{n} y+z^{2}+t^{3}+x p(x)$ and $x^{n} y+p(x)\left(z^{2}+t^{3}+x\right)$ are equivalent by an automorphism of $\mathbb{C}[x, y, z, t, w]$, whereas they are not equivalent up to an automorphism of $\mathbb{C}[x, y, z, t]$. Their zero-sets are even nonisomorphic smooth affine threefolds.

As a very particular case of the above discussion, we obtain the result announced in the introduction, namely:

Corollary 1.6. The smooth contractible affine threefolds

$$
X_{a}=\left\{x^{4} y+z^{2}+t^{3}+x+x^{2}+a x^{3}=0\right\}, a \in \mathbb{C},
$$

are pairwise nonisomorphic. However, their cylinders $X_{a} \times \mathbb{A}^{1}, a \in \mathbb{C}$, are all isomorphic.

Remark 1.7. Let $W \subset \mathbb{A}^{4} \times \mathbb{A}^{1}=\operatorname{Spec}(\mathbb{C}[x, y, z, t][a])$ be the smooth variety with equation $x^{4} y+z^{2}+t^{3}+x+x^{2}+a x^{3}=0$. The projection on the second factor equips $W$ with the structure of a one-parameter flat family $\pi: W \rightarrow \mathbb{A}^{1}$ of affine threefolds, with closed fibers isomorphic to the varieties $X_{a}, a \in \mathbb{C}$, above. It follows from the construction given in the proof of Theorem 1.3 that $\pi: W \rightarrow \mathbb{A}^{1}$ 
is holomorphically trivial. Namely, letting $f=1+\left(a-\frac{1}{2}\right) x-\left(a-\frac{1}{3}\right) x^{2}$ and $h=x^{-4}\left(\exp (x f(x))-\left(1+x+a x^{2}\right)\right)$, the biholomorphism of $\mathbb{A}^{4} \times \mathbb{A}^{1}$ defined by

$$
(x, y, z, t, a) \mapsto\left(x, y-h(x)\left(z^{2}+t^{3}\right), \exp \left(\frac{1}{2} x f(x)\right) z, \exp \left(\frac{1}{3} x f(x)\right) t, a\right)
$$

maps the family $\tilde{W} \subset \mathbb{A}^{4} \times \mathbb{A}^{1}$ with equation $x^{4} y+\left(1+x+a x^{2}\right)\left(z^{2}+t^{3}+x\right)=0$ biholomorphically onto $W$. Since the ideals $\left(x^{4},\left(1+x+a x^{2}\right)\left(z^{2}+t^{3}+x\right)\right)$ and $\left(x^{4}, z^{2}+t^{3}+x\right)$ of $\mathbb{C}[x, y, z, t][a]$ are equal, Lemma 1.1 implies that $\tilde{W}$ is isomorphic as a flat family over $\mathbb{A}^{1}$ to the trivial one $V_{1} \times \mathbb{A}^{1}$, where

$$
V_{1}=\left\{x^{4} y+z^{2}+t^{3}+x=0\right\} \subset \mathbb{A}^{4} .
$$

Summing up, $\pi: W \rightarrow \mathbb{A}^{1}$ is a holomorphically trivial family of pairwise nonisomorphic exotic algebraic structures on $\mathbb{A}^{3}$. This answers Problem 3 in [29.

\section{A GeOMEtRic interpretation}

Here we give a geometric interpretation of the existence of an isomorphism between the cylinders over the varieties $X_{a}, a \in \mathbb{C}$, in terms of a variant of the famous Danielewski fiber product trick [4. Of course, the construction below can be adapted to cover the general case, but we find it more enlightening to only consider the particular case of the varities $X_{0}$ and $X_{1}$ in $\mathbb{A}^{4}=\operatorname{Spec}(\mathbb{C}[x, y, z, t])$ defined respectively by the equations

$$
x^{4} y+z^{2}+t^{3}+x+x^{2}=0 \text { and } x^{4} y+z^{2}+t^{3}+x+x^{2}+x^{3}=0 .
$$

For our purpose, it is convenient to use the fact that $X_{0}$ and $X_{1}$ are isomorphic to the varieties $X$ and $Y$ in $\mathbb{A}^{4}$ defined respectively by the equations

$$
x^{4} z=y^{2}+x+x^{2}-t^{3} \quad \text { and } \quad x^{4} z=\left(1+\alpha x^{2}\right) y^{2}+x+x^{2}-t^{3},
$$

where $\alpha=-\frac{5}{3}$. Clearly, the first isomorphism is simply induced by the coordinate change $(x, y, z, t) \mapsto(x, z,-y,-t)$. For the second isomorphism, one checks first that for $\beta=-1 / 3$, the $\mathbb{C}[x]$-automorphism of $\mathbb{C}[x][z, t]$ defined by the matrix

$$
\left(\begin{array}{cc}
1-\beta x^{2}+\frac{1}{2} \beta^{2} x^{4} & \frac{1}{2} \beta^{2} x^{4} \\
\frac{1}{2} \beta^{2} x^{4} & 1+\beta x^{2}+\frac{1}{2} \beta^{2} x^{4}
\end{array}\right) \in \mathrm{GL}_{2}(\mathbb{C}[x])
$$

maps the ideal $\left(x^{4},\left(1+\alpha x^{2}\right) z^{2}+x+x^{2}+t^{3}\right)$ of $\mathbb{C}[x][z, t]$ isomorphically onto the one $\left(x^{4}, z^{2}+x+x^{2}+x^{3}+t^{3}\right)$. By virtue of Lemma 1.1, the corresponding automorphism of $\mathbb{A}^{3}$ lifts to an isomorphism between $X_{1}$ and the subvariety of $\mathbb{A}^{4}$ defined by the equation $x^{4} y+\left(1+\alpha x^{2}\right) z^{2}+x+x^{2}+t^{3}=0$, and so, we eventually get the desired isomorphism by composing with the previous coordinate change.

2.1. Now, the principle is the following: we observe that both $X$ and $Y$ come equipped with $\mathbb{G}_{a}$-actions induced by the ones on $\mathbb{A}^{4}$ associated respectively with the locally nilpotent derivations $x^{4} \partial_{y}+2 y \partial_{z}$ and $x^{4} \partial_{y}+2\left(1+\alpha x^{2}\right) y \partial_{z}$ of $\mathbb{C}[x, y, z, t]$. The latter restrict to free actions on the open subsets $X^{*}=X \backslash\{x=t=0\}$ and $Y^{*}=Y \backslash\{x=t=0\}$ of $X$ and $Y$ respectively, and so, they admit quotients $X^{*} \rightarrow X^{*} / \mathbb{G}_{a}$ and $Y^{*} \rightarrow Y^{*} / \mathbb{G}_{a}$ in the form of étale locally trivial $\mathbb{G}_{a}$-bundles over suitable algebraic spaces. We first check that $X^{*} / \mathbb{G}_{a}$ and $Y^{*} / \mathbb{G}_{a}$ are isomorphic to a same algebraic space $\mathfrak{S}$. This implies that the fiber product $W=X^{*} \times_{\mathfrak{S}} Y^{*}$ has the structure of a locally trivial $\mathbb{G}_{a}$-bundle over both $X^{*}$ and $Y^{*}$ via the first and the second projection respectively. Since $X^{*}$ and $Y^{*}$ are both strictly quasi-affine, there is no guarantee a priori that these $\mathbb{G}_{a}$-bundles are trivial. However, we check 
below that it is indeed the case. Therefore, since $X$ and $Y$ are affine, normal, and $X \backslash X^{*}$ and $Y \backslash Y^{*}$ have codimension 2 in $X$ and $Y$ respectively, the corresponding isomorphism $X^{*} \times \mathbb{A}^{1} \simeq W \simeq Y^{*} \times \mathbb{A}^{1}$ extends to one of $X \times \mathbb{A}^{1} \simeq Y \times \mathbb{A}^{1}$.

2.2. Let us check first that the quotient spaces $X^{*} / \mathbb{G}_{a}$ and $Y^{*} / \mathbb{G}_{a}$ are indeed isomorphic. The restriction of the projection $\operatorname{pr}_{x, t}: \mathbb{A}^{4} \rightarrow \mathbb{A}^{2}=\operatorname{Spec}(\mathbb{C}[x, t])$ to $X^{*}$ and $Y^{*}$ induces $\mathbb{G}_{a}$-invariant morphisms $\alpha: X^{*} \rightarrow \mathbb{A}^{2} \backslash\{(0,0)\}$ and $\beta$ : $Y^{*} \rightarrow \mathbb{A}^{2} \backslash\{0,0\}$. The latter restrict to trivial $\mathbb{G}_{a}$-bundles over $\mathbb{A}^{2} \backslash\{x=0\}$. In contrast, the fiber of each morphism over a closed point $(0, t) \in \mathbb{A}^{2} \backslash\{(0,0)\}$ consists of the disjoint union of two affine lines $\{x=0, y= \pm \mu\}$ where $\mu$ is a square root of $t^{3}$, whereas the fiber over the nonclosed point $(x) \in \mathbb{C}[x, t]$ with residue field $\mathbb{C}(t)$ corresponding to the punctured line $\{x=0\} \subset \mathbb{A}^{2} \backslash\{(0,0)\}$ is isomorphic to the affine line over the degree 2 Galois extension $\mathbb{C}(t)[y] /\left(y^{2}-t^{3}\right)$ of $\mathbb{C}(t)$. This indicates that the quotient spaces $X^{*} / \mathbb{G}_{a}$ and $Y^{*} / \mathbb{G}_{a}$ should be obtained from $\mathbb{A}^{2} \backslash\{(0,0)\}$ by replacing the punctured line $\{x=0\}$ by a nontrivial double étale covering of itself. An algebraic space $\mathfrak{S}$ with this property can be constructed in two steps as follows: first we let $U_{\lambda}=\operatorname{Spec}\left(\mathbb{C}\left[x, \lambda^{ \pm 1}\right]\right), U_{\lambda \lambda}=\operatorname{Spec}\left(\mathbb{C}\left[x^{ \pm 1}, \lambda^{ \pm 1}\right]\right)$, and we define an algebraic space $\mathfrak{S}_{\lambda}$ as the quotient of $U_{\lambda}$ by the following étale equivalence relation:

$$
(s, t): R_{\lambda}=U_{\lambda} \sqcup U_{\lambda \lambda} \longrightarrow U_{\lambda} \times U_{\lambda}, \begin{cases}U_{\lambda} \ni(x, \lambda) & \mapsto((x, \lambda),(x, \lambda)), \\ U_{\lambda \lambda} \ni(x, \lambda) & \mapsto((x, \lambda),(x,-\lambda)) .\end{cases}
$$

By construction, the $R_{\lambda}$-invariant morphism $U_{\lambda} \rightarrow \operatorname{Spec}\left(\mathbb{C}\left[x, t^{ \pm 1}\right]\right),(x, \lambda) \mapsto$ $\left(x, \lambda^{2}\right)$ descends to a morphism $\mathfrak{S}_{\lambda} \rightarrow \operatorname{Spec}\left(\mathbb{C}\left[x, t^{ \pm 1}\right]\right)$ restricting to an isomorphism over $\operatorname{Spec}\left(\mathbb{C}\left[x^{ \pm 1}, t^{ \pm 1}\right]\right)$. The fiber over the punctured line $\{x=0\}$ is isomorphic to $\operatorname{Spec}\left(\mathbb{C}(t)[\lambda] /\left(\lambda^{2}-t^{3}\right)\right)$. Now we let $\mathfrak{S}$ be the algebraic space obtained by gluing $\mathfrak{S}_{\lambda}$ and $U_{x}=\operatorname{Spec}\left(\mathbb{C}\left[x^{ \pm 1}, t\right]\right)$ by the identity on $\operatorname{Spec}\left(\mathbb{C}\left[x^{ \pm 1}, t^{ \pm 1}\right]\right)$. By construction, $\mathfrak{S}$ comes equipped with an étale cover $p: V \rightarrow \mathfrak{S}$ by the scheme $V=U_{x} \sqcup U_{\lambda}$. We let $U_{x, \lambda}=U_{x} \times_{\mathfrak{S}} U_{\lambda} \simeq \operatorname{Spec}\left(\mathbb{C}\left[x^{ \pm 1}, \lambda^{ \pm 1}\right]\right)$.

Lemma 2.3. The quotient spaces $X^{*} / \mathbb{G}_{a}$ and $Y^{*} / \mathbb{G}_{a}$ are both isomorphic to $\mathfrak{S}$.

Proof. The argument is very similar to the one used in [5].

1) The case of $X^{*}$. This quasi-affine threefold is covered by two $\mathbb{G}_{a}$-invariant open subsets

$$
V_{x}=X^{*} \backslash\{x=0\}=X \backslash\{x=0\} \quad \text { and } \quad V_{t}=X^{*} \backslash\{t=0\}=X \backslash\{t=0\} .
$$

Letting $U_{x}=\operatorname{Spec}\left(\mathbb{C}\left[x^{ \pm 1}, t\right]\right)$, one easily checks that the morphism

$$
U_{x} \times \mathbb{G}_{a} \longrightarrow V_{x},(x, t, v) \mapsto\left(x, x^{4} v, x^{4} v^{2}+x^{-4}\left(-t^{3}+x+x^{2}\right), t\right)
$$

is an isomorphism, equivariant for the $\mathbb{G}_{a}$-action on $U_{x} \times \mathbb{G}_{a}$ by translations on the second factor, which yields a trivialization of the induced $\mathbb{G}_{a}$-action on $V_{x}$. In contrast, the induced action on $V_{t}$ is not trivial. However, letting $U_{\lambda}=\operatorname{Spec}\left(\mathbb{C}\left[x, \lambda^{ \pm 1}\right]\right)$, we claim that there exists $\sigma, \xi \in \mathbb{C}\left[x, \lambda^{ \pm 1}\right]$ such that the morphism

$$
U_{\lambda} \times \mathbb{G}_{a} \longrightarrow V_{t},(x, \lambda, v) \mapsto\left(x, x^{4} v+\sigma,\left(x^{4} v+2 \sigma\right) v+\xi, \lambda^{2}\right)
$$

is étale and equivariant for the $\mathbb{G}_{a}$-action on $U_{\lambda} \times \mathbb{G}_{a}$ by translations on the second factor, and hence defines an étale trivialization on the induced action on $V_{t}$. This can be seen as follows: let

$$
V_{\lambda}=V_{t} \times_{\mathbb{A}_{*}^{1}} \mathbb{A}_{*}^{1} \simeq \operatorname{Spec}\left(\mathbb{C}\left[x, y, z, \lambda^{ \pm 1}\right] /\left(x^{4} z-y^{2}+\lambda^{6}-x-x^{2}\right)\right)
$$


be the pull-back of $V_{t}$ by the Galois covering

$$
\varphi: \mathbb{A}_{*}^{1}=\operatorname{Spec}\left(\mathbb{C}\left[\lambda^{ \pm 1}\right]\right) \rightarrow \mathbb{A}_{*}^{1}=\operatorname{Spec}\left(\mathbb{C}\left[t^{ \pm 1}\right]\right), \lambda \mapsto t=\lambda^{2} .
$$

Since $\lambda \in \mathbb{C}\left[x, \lambda^{ \pm 1}\right]$ is invertible it follows that one can find $\sigma \in \mathbb{C}\left[x, \lambda^{ \pm 1}\right]$ with $\operatorname{deg}_{x} \sigma \leq 3$ and $\sigma(0, \lambda)=\lambda^{3}$, and $\xi \in \mathbb{C}\left[x, \lambda^{ \pm 1}\right]$ such that

$$
y^{2}-\lambda^{6}+x+x^{2}=(y-\sigma)(y+\sigma)+x^{4} \xi .
$$

Note that $\sigma$ and $\xi$, considered as Laurent polynomials in the variable $\lambda$, are necessarily odd and even respectively. This identity implies in turn that $V_{\lambda}$ is isomorphic to the subvariety of $\operatorname{Spec}\left(\mathbb{C}\left[x, y, z^{\prime}, \lambda^{ \pm 1}\right]\right)$ defined by the equation $x^{4} z^{\prime}=(y-\sigma)(y+\sigma)$, where $z^{\prime}=z-\xi$. The $\mathbb{G}_{a}$-action on $V_{t}$ lifts to the one on $V_{\lambda}$ induced by the locally nilpotent derivation $x^{4} \partial_{y}+2 y \partial_{z^{\prime}}$. The open subset $V_{\lambda+}=V_{\lambda} \backslash\{x=y+\sigma=0\} \simeq \operatorname{Spec}\left(\mathbb{C}\left[x, \lambda^{ \pm 1}\right][v]\right)$, where

$$
v=\left.x^{-4}(y-\sigma)\right|_{V_{\lambda+}}=\left.(y+\sigma)^{-1} z^{\prime}\right|_{V_{\lambda+}},
$$

is equivariantly isomorphic to $U_{\lambda} \times \mathbb{G}_{a}$, where $\mathbb{G}_{a}$ acts on the second factor by translations, and the restriction of the étale morphism $\mathrm{pr}_{1}: V_{t} \times_{\mathbb{A}_{*}^{1}} \mathbb{A}_{*}^{1} \rightarrow V_{t}$ to $V_{\lambda} \backslash\{x=y+\sigma=0\} \simeq U_{\lambda} \times \mathbb{G}_{a}$ yields the expected étale trivialization. It follows from this description that $X^{*} / \mathbb{G}_{a}$ is isomorphic to an algebraic space obtained as the quotient of disjoint unions of $U_{x}=V_{x} / \mathbb{G}_{a}$ and $U_{\lambda}=V_{\lambda+} / \mathbb{G}_{a}$ by a certain étale equivalence relation. Clearly, the only nontrivial part is to check that $V_{t} / \mathbb{G}_{a}$ is isomorphic to the algebraic space $\mathfrak{S}_{\lambda}$ of subsection 2.2 above. In view of I.5.8 in [15] it is enough to show that we have a cartesian square

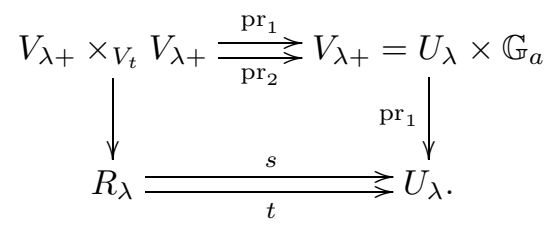

Letting $g(x, \lambda, v)=x^{4} v+\sigma(x, \lambda) \in \mathbb{C}\left[x, \lambda^{ \pm 1}, v\right]$ and $h=\left(x^{4} v+2 \sigma(x, \lambda)\right) v+$ $\xi(x, \lambda) \in \mathbb{C}\left[x, \lambda^{ \pm 1}, v\right], V_{\lambda+} \times_{V_{t}} V_{\lambda+}$ is isomorphic to the spectrum of the ring

$$
\begin{aligned}
A=\mathbb{C}\left[x, \lambda^{ \pm 1}, \lambda_{1}^{ \pm 1}, v, v_{1}\right] / & \\
& \left(g(x, \lambda, v)-g\left(x, \lambda_{1}, v_{1}\right), h(x, \lambda, v)-h\left(x, \lambda_{1}, v_{1}\right), \lambda^{2}-\lambda_{1}^{2}\right) .
\end{aligned}
$$

Since $\lambda$ is invertible and $\sigma(0, \lambda)=\lambda^{3}, x$ and $\sigma$ generate the unit ideal in $\mathbb{C}\left[x, \lambda^{ \pm 1}\right]$. It follows that $A$ decomposes as the direct product of the rings

$$
\begin{aligned}
A_{0} & =\mathbb{C}\left[x, \lambda^{ \pm 1}, v, v_{1}\right] /\left(g(x, \lambda, v)-g\left(x, \lambda, v_{1}\right), h(x, \lambda, v)-h\left(x, \lambda, v_{1}\right)\right) \\
& \simeq \mathbb{C}\left[x, \lambda^{ \pm 1}, v, v_{1}\right] /\left(x^{4}\left(v-v_{1}\right), x^{4}\left(v^{2}-v_{1}^{2}\right)+2 \sigma(x, \lambda)\left(v-v_{1}\right)\right) \\
& \simeq \mathbb{C}\left[x, \lambda^{ \pm 1}, v, v_{1}\right] /\left(x^{4}\left(v-v_{1}\right), 2 \sigma(x, \lambda)\left(v-v_{1}\right)\right) \\
& \simeq \mathbb{C}\left[x, \lambda^{ \pm 1}\right][v]
\end{aligned}
$$


and

$$
\begin{aligned}
A_{1} & =\mathbb{C}\left[x, \lambda^{ \pm 1}, v, v_{1}\right] /\left(g(x, \lambda, v)-g\left(x,-\lambda, v_{1}\right), h(x, \lambda, v)-h\left(x,-\lambda, v_{1}\right)\right) \\
& \simeq \mathbb{C}\left[x, \lambda^{ \pm 1}, v, v_{1}\right] /\left(x^{4}\left(v-v_{1}\right)+2 \sigma(x, \lambda), x^{4}\left(v^{2}-v_{1}^{2}\right)+2 \sigma(x, \lambda)\left(v+v_{1}\right)\right) \\
& \simeq \mathbb{C}\left[x, \lambda^{ \pm 1}, v, v_{1}\right] /\left(x^{4}\left(v-v_{1}\right)+2 \sigma(x, \lambda)\right) \\
& \simeq \mathbb{C}\left[x^{ \pm 1}, \lambda^{ \pm 1}, v, v_{1}\right] /\left(x^{4}\left(v-v_{1}\right)+2 \sigma(x, \lambda)\right) \\
& \simeq \mathbb{C}\left[x^{ \pm 1}, \lambda^{ \pm 1}\right][v] .
\end{aligned}
$$

Thus $V_{\lambda+} \times_{V_{t}} V_{\lambda+}$ is isomorphic to $R_{\lambda} \times \mathbb{A}^{1}$ and the above diagram is clearly cartesian. This completes the proof for $X^{*}$.

2) The case of $Y^{*}$. Similarly as for the case of $X^{*}, Y^{*}$ is covered by two $\mathbb{G}_{a^{-}}$ invariant open subsets

$$
W_{x}=Y^{*} \backslash\{x=0\}=Y \backslash\{x=0\} \quad \text { and } \quad W_{t}=Y^{*} \backslash\{t=0\}=Y \backslash\{t=0\},
$$

and the morphism

$$
U_{x} \times \mathbb{G}_{a} \longrightarrow W_{x},(x, t, v) \mapsto\left(x, x^{4} v, x^{4}\left(1+\alpha x^{2}\right) v^{2}+x^{-4}\left(-t^{3}+x+x^{2}\right), t\right)
$$

defines a trivialization of the induced $\mathbb{G}_{a}$-action on $W_{x}$. To obtain an étale trivialization of the $\mathbb{G}_{a}$-action on $W_{t}$, one checks first that there exists $\zeta \in \mathbb{C}\left[x, \lambda^{ \pm 1}\right]$ such that for

$$
\tau=\left(1-\frac{1}{2} \alpha x^{2}\right) \sigma(x, \lambda)
$$

the identity

$$
\left(1+\alpha x^{2}\right) y^{2}-\lambda^{6}+x+x^{2}=\left(1+\alpha x^{2}\right)(y-\tau)(y+\tau)+x^{4} \zeta(x, \lambda)
$$

holds in $\mathbb{C}\left[x, \lambda^{ \pm 1}, y\right]$. Then one checks in a similar way as above that the morphism

$$
U_{\lambda} \times \mathbb{G}_{a} \longrightarrow W_{t},(x, \lambda, v) \mapsto\left(x, x^{4} v+\tau,\left(x^{4} v+2 \tau\right) v+\zeta, \lambda^{2}\right)
$$

yields an étale trivialization, that $W_{t} / \mathbb{G}_{a} \simeq U_{\lambda} / R_{\lambda}=\mathfrak{S}_{\lambda}$ and that $Y^{*} / \mathbb{G}_{a} \simeq \mathfrak{S}$.

2.4. From now on, we identify $X^{*} / \mathbb{G}_{a}$ and $Y^{*} / \mathbb{G}_{a}$ with the algebraic space $\mathfrak{S}$ constructed in subsection 2.2 above, and we let $W=X^{*} \times_{\mathfrak{S}} Y^{*}$. By construction, $W$ is a scheme, equipped with a structure of a Zariski locally trivial $\mathbb{G}_{a}$-bundle over $X^{*}$ and $Y^{*}$ via the first and the second projection, respectively. The following completes the proof.

Lemma 2.5. We have isomorphisms $X^{*} \times \mathbb{A}^{1} \simeq W \simeq Y^{*} \times \mathbb{A}^{1}$.

Proof. We will show more precisely that the $\mathbb{G}_{a}$-bundles $\mathrm{pr}_{1}: W \rightarrow X^{*}$ and $\mathrm{pr}_{2}$ : $W \rightarrow Y^{*}$ are both trivial. It follows from the description of the étale trivialization given in the proof of Lemma 2.3 above that the isomorphy class of the $\mathbb{G}_{a}$-bundle $X^{*} \rightarrow \mathfrak{S}$ in $H_{\text {ét }}^{1}\left(\mathfrak{S}, \mathcal{O}_{\mathfrak{S}}\right)$ is represented by the Čech 1-cocycle

$$
\left(x^{-4} \sigma, 2 x^{-4} \sigma\right) \in \Gamma\left(U_{x, \lambda}, \mathcal{O}_{U_{x, \lambda}}\right) \times \Gamma\left(U_{\lambda \lambda}, \mathcal{O}_{U_{\lambda \lambda}}\right)=\mathbb{C}\left[x^{ \pm 1}, \lambda^{ \pm 1}\right]^{2}
$$

with value in $\mathcal{O}_{\mathfrak{S}}$ for the étale cover $p: V \rightarrow \mathfrak{S}$ of $\mathfrak{S}$. Similarly, the isomorphy class of the $\mathbb{G}_{a}$-bundle $Y^{*} \rightarrow \mathfrak{S}$ is represented by the Cech 1-cocycle

$$
\left(x^{-4} \tau, 2 x^{-4} \tau\right) \in \Gamma\left(U_{x, \lambda}, \mathcal{O}_{U_{x, \lambda}}\right) \times \Gamma\left(U_{\lambda \lambda}, \mathcal{O}_{U_{\lambda \lambda}}\right)=\mathbb{C}\left[x^{ \pm 1}, \lambda^{ \pm 1}\right]^{2} .
$$


This implies in turn that the isomorphy class of the $\mathbb{G}_{a}$-bundle $\operatorname{pr}_{1}: W \rightarrow X^{*}$ in $H_{\text {êt }}^{1}\left(X^{*}, \mathcal{O}_{X^{*}}\right)$ is represented by the Cech 1-cocyle

$$
\alpha=\left(x^{-4} \tau, 2 x^{-4} \tau\right) \in \Gamma\left(\mathbb{A}_{U_{x, \lambda}}^{1}, \mathcal{O}_{\mathbb{A}_{U_{x}, \lambda}^{1}}\right) \times \Gamma\left(\mathbb{A}_{U_{\lambda \lambda}}^{1}, \mathcal{O}_{\mathbb{A}_{U_{\lambda \lambda}}^{1}}\right)=\left(\mathbb{C}\left[x^{ \pm 1}, \lambda^{ \pm 1}\right][v]\right)^{2}
$$

with value in $\mathcal{O}_{X^{*}}$ for the étale cover given by $U_{x} \times \mathbb{G}_{a}$ and $U_{\lambda} \times \mathbb{G}_{a}$. By definition, $\operatorname{pr}_{1}: W \rightarrow X^{*}$ is a trivial $\mathbb{G}_{a}$-bundle if and only if $\alpha$ is a coboundary. This is the case if and only if there exists

$$
\beta_{x} \in \Gamma\left(\mathbb{A}_{U_{x}}^{1}, \mathcal{O}_{\mathbb{A}_{U_{x}}^{1}}\right)=\mathbb{C}\left[x^{ \pm 1}, t\right][v] \quad \text { and } \quad \beta_{\lambda} \in \Gamma\left(\mathbb{A}_{U_{\lambda}}^{1}, \mathcal{O}_{\mathbb{A}_{U_{\lambda}}^{1}}\right)=\mathbb{C}\left[x, \lambda^{ \pm 1}\right][v]
$$

such that

$$
\left\{\begin{array}{l}
x^{-4} \tau=\beta_{\lambda}\left(x, \lambda, v-x^{-4} \sigma\right)-\beta_{x}\left(x, \lambda^{2}, v\right), \\
2 x^{-4} \tau=\beta_{\lambda}(x, \lambda, v)-\beta_{\lambda}\left(x,-\lambda, v+2 x^{-4} \sigma\right) .
\end{array}\right.
$$

Since

$$
\tau(x, \lambda)=\left(1-\frac{1}{2} \alpha x^{2}\right) \sigma(x, \lambda),
$$

one can choose for instance

$$
\beta_{x}(x, t, v)=-\left(1-\frac{1}{2} \alpha x^{2}\right) v \quad \text { and } \quad \beta_{\lambda}(x, \lambda, v)=-\left(1-\frac{1}{2} \alpha x^{2}\right) v .
$$

The fact that $\mathrm{pr}_{2}: W \rightarrow Y^{*}$ is also a trivial $\mathbb{G}_{a}$-bundle follows from a similar argument using the identity

$$
\sigma(x, \lambda)=\left(1+\frac{1}{2} \alpha x^{2}\right) \tau(x, \lambda)+\frac{1}{4} \alpha^{2} x^{4} \sigma(x, \lambda) .
$$

\section{REFERENCES}

[1] S. Abhyankar, P. Eakin, and W. Heinzer, On the uniqueness of the coefficient ring in a polynomial ring, J. Algebra 23 (1972), 310-342. MR0306173 (46:5300)

[2] A. Choudary and A. Dimca, Complex hypersurfaces diffeomorphic to affine spaces, Kodai Math. J. 17, no. 2 (1994), 171-178. MR1282208 (95f:14084)

[3] A. J. Crachiola and L. Makar-Limanov, An algebraic proof of a cancellation theorem for surfaces, J. Algebra 320 (2008), no. 8, 3113-3119. MR2450715 (2009h:14105)

[4] W. Danielewski, On a cancellation problem and automorphism groups of affine algebraic varieties, preprint, Warsaw, 1989.

[5] A. Dubouloz, The cylinder over the Koras-Russell cubic threefold has a trivial MakarLimanov invariant, Transformation Groups 14, no. 3 (2009), 531-539. MR2534798

[6] A. Dubouloz, Additive group actions on Danielewski varieties and the Cancellation Problem, Math. Z. 255 (2007), no. 1, 77-93. MR2262722(2007i:14066)

[7] A. Dubouloz, L. Moser-Jauslin and P.-M. Poloni, Inequivalent embeddings of the KorasRussell cubic threefold, Michigan Math. J. 59 (2010), 531-539.

[8] K.H. Fieseler, On complex affine surfaces with $\mathbb{C}_{+}$-actions, Comment. Math. Helvetici 69 (1994), 5-27. MR1259603 (95b:14027)

[9] D. Finston and S. Maubach, The automorphism group of certain factorial threefolds and a cancellation problem, Israel J. Math. 163 (2008), 369-381. MR2391136 (2009a:14078)

[10] M. Hochster, Nonuniqueness of coefficient rings in a polynomial ring, Proc. Amer. Math. Soc. 34 (1972), 81-82. MR0294325 (45:3394)

[11] S. Iitaka and T. Fujita, Cancellation theorem for algebraic varieties, J. Fac. Sci. Univ. Tokyo 24 (1977), 123-127. MR0450278 (56:8574)

[12] Z. Jelonek, On the cancellation problem, Math. Ann. 344 (2009), 769-778. MR2507623 (2010f:14066)

[13] S. Kaliman and L. Makar-Limanov, AK-invariant of affine domains, in Affine Algebraic Geometry, Osaka Univ. Press, Osaka, 2007, 231-255. MR2327241 (2008f:14076)

[14] S. Kaliman and M. Zaidenberg, Affine modifications and affine varieties with a very transitive automorphism group, Transform. Groups 4 (1999), 53-95. MR.1669174 (2000f:14099) 
[15] D. Knutson, Algebraic spaces, Lecture Notes in Math., vol. 203, Springer Verlag, 1971. MR0302647 (46:1791)

[16] L. Makar-Limanov, On the hypersurface $x+x^{2} y+z^{2}+t^{3}=0$ in $\mathbb{C}^{4}$ or a $\mathbb{C}^{3}$-like threefold which is not $\mathbb{C}^{3}$, Israel J. Math. 96 (1996), 419-429. MR.1433698(98a:14052)

[17] L. Makar-Limanov, P. van Rossum, V. Shpilrain and J.-T. Yu, The stable equivalence and cancellation problems, Comment. Math. Helv. 79 (2004), 341-349. MR2059436(2005d:14094)

[18] M. Miyanishi, An algebraic characterization of the affine plane, J. Math. Kyoto Univ. 15 (1975), 169-184. MR0419460 (54:7481)

[19] M. Miyanishi and T. Sugie, Affine surfaces containing cylinderlike open sets, J. Math. Kyoto Univ. 20 (1980), 11-42. MR564667 (81h:14020)

[20] L. Moser-Jauslin, Automorphism groups of Koras-Russell threefolds of the first kind, to appear in "Affine Algebraic Geometry: A Conference in Honour of Peter Russell", Montreal, 1-5 June 2009, CRM Proceedings Lecture Notes.

[21] L. Moser-Jauslin and P.-M. Poloni, Embeddings of a family of Danielewski hypersurfaces and certain $\mathbb{C}_{+}$-actions on $\mathbb{C}^{3}$, Ann. Inst. Fourier (Grenoble) 56, no. 5 (2006), 1567-1581. MR.2273864 (2007k:14127)

[22] M. P. Murthy, Cancellation problem for projective modules over certain affine algebras, Proceedings of the International Colloquium on Algebra, Arithmetic and Geometry Mumbai, Narosa Publishing House, 2000, 493-507. MR1940679 (2003j:13010)

[23] T. Sugie, On a characterization of surfaces containing cylinderlike open sets, Osaka J. Math. 17 (1980), 363-376. MR.587756 (82c:14026)

[24] T. Sugie, Algebraic characterization of the affine plane and the affine 3-space, Topological Methods in Algebraic Transformation Groups (H. Kraft et al., eds.). Progress in Math., vol. 80, Birkhäuser Verlag, Basel - Boston, 1989, 177-190. MR.1040864 (91a:14022)

[25] J. Wilkens, On the cancellation problem for surfaces, C. R. Acad. Sci. Paris Ser. I Math. 326 (1998), no. 9, 1111-1116. MR.1647227 (99i:14040)

[26] A. van den Essen, S. Maubach, and S. Vénéreau, The special automorphism group of $R[t] /\left(t^{m}\right)\left[x_{1} \ldots, x_{n}\right]$ and coordinates of a subring of $R[t]\left[x_{1}, \ldots, x_{n}\right]$, J. Pure Appl. Algebra 210(1) (2007), 141-146. MR.2311177 (2008g:14119)

[27] M. Zaidenberg, An analytic cancellation theorem and exotic algebraic structures on $\mathbb{C}^{n}$, $n \geq 3$, Astérisque 217 (1993), 251-282. MR.1247763 (94k:32045)

[28] M. Zaidenberg, On exotic algebraic structures on affine spaces, Algebra and Analysis. St. Petersbourg Math. J. 10 (1999), no. 5, 3-73. MR.1734345 (2001d:14069)

[29] M. Zaidenberg, Selected Problems, preprint arXiv:math/0501457v1, 2005.

Institut de Mathématiques de Bourgogne, Université de Bourgogne, 9 avenue Alain SAVARY - BP 47870, 21078 Dijon CEDEx, France

E-mail address: Adrien.Dubouloz@u-bourgogne.fr

Institut de Mathématiques de Bourgogne, Université de Bourgogne, 9 avenue Alain SAVARY - BP 47870, 21078 Dijon Cedex, France

E-mail address: moser@u-bourgogne.fr

Mathematisches Institut, Universitat Basel, Rheinsprung 21, CH-4051 Basel, SwitzerLAND

E-mail address: pierre-marie.poloni@unibas.ch 